\title{
A computational framework for the analysis of rain-induced erosion in wind turbine blades, part II: drop impact-induced stresses and blade coating fatigue life
}

\author{
B. Amirzadeh ${ }^{1}$, A. Louhghalam ${ }^{1}$, M. Raessi ${ }^{1}$, M. Tootkaboni ${ }^{1, *}$ \\ University of Massachusetts Dartmouth, 285 Old Westport Rd. North Dartmouth MA, USA
}

\begin{abstract}
With the current trend in wind energy production, the need to manufacture larger wind turbine blades is on the rise. The high blade tip velocities associated with large blades subject them to various damages due to high speed impact with foreign objects such as raindrops. This paper is the second part in a two-part paper that presents a framework for rain erosion prediction in wind turbine blades. In part I, a stochastic rain texture model and multi-resolution simulation of raindrop impact on solid object were discussed. In part II, the predicted impact pressure profiles are imported into a finite element model of the wind turbine blade shell to analyze the raindrop impact-induced transient stresses within the blade coating and the ensuing fatigue damage pattern. The analysis is complemented with a fatigue stress-life estimation process that integrates elements of fatigue life calculation with 3D fields of raindrops generated from the stochastic rain texture model to relate damage accumulation rates to rain intensities. These accumulation rates, together with the statistics of rainfall history, provide a means for estimating the expected fatigue life of the blade coating as an indication of the onset of surface roughening or the end of the incubation period.

Keywords: wind turbine blade, rain-induced erosion, fatigue analysis, damage accumulation rate, expected fatigue life
\end{abstract}

\section{Introduction}

In the past decade, there has been a ten-fold increase in the amount of power generated from wind providing $2.5 \%$ of the total energy in the world as of 2012 [1]. It is anticipated that, by the end of this decade, this number will increase to as high as 8 to $12 \%$ [2] driven by the production of wind turbines with higher power output. In fact this trend has already begun and the 8 MW Vestas V164 [3] and the 7 MW Samsung S7.0-171 [4] wind turbines are such examples. These wind turbines have rotor diameters in the range of 170 meters providing the evidence that higher power output usually comes with larger rotor blades. Wind turbine blades are currently the largest composite structures manufactured in series [5]. Considering the length of these blades and a typical angular velocity of 12

\footnotetext{
${ }^{*}$ Corresponding author

Email addresses: bamirzadeh@umassd.edu (B. Amirzadeh), alouhghalam@umassd.edu (A. Louhghalam), mraessi@umassd.edu (M. Raessi), mtootkaboni@umassd.edu (M. Tootkaboni)

${ }^{1}$ University of Massachusetts Dartmouth

Preprint submitted to Journal of Wind Engineering E Industrial Aerodynamics

November 18, 2016
} 
revolutions per minute, blade tip velocities in excess of $100 \mathrm{~m} / \mathrm{s}$ are not unusual. In addition, most wind turbines are installed in locations where the environmental conditions are detrimental to the turbine blade's surface [6,7]. Although a matrix rich layer of gelcoat is often applied to the blades' outer surface for protection [8], some of these environmental conditions, together with high blade tip velocities bring about susceptibility to erosion and damage induced by foreign object impact. Rain-induced erosion of the blades begins with a gradual increase in blade's surface roughness [9] which subsequently increases the aerodynamic drag coefficient of the blades, resulting in an undesirable lower performance and energy loss. For instance, in simulated erosion experiments for a $2.5 \mathrm{MW}$ wind turbine blade, it has been reported that the drag coefficient can increase from 6 to $500 \%$ depending on the severity of erosion, accompanied by up to 0.17 loss in the lift coefficient. This can lead up to $25 \%$ annual energy loss for the wind turbine [9]. Furthermore, surface roughness can affect the dynamic stall characteristics and aerodynamic forces $[10,11,12]$ which are known to play an important role in turbine's aerodynamic performance $[13,14]$ and the severity and nature of flow-induced vibrations [15].

In addition, it has been reported that pre-erosion by liquid drops can accelerate subsequent erosion by solid particles [16] which further deteriorates the turbines' performance. Signs of leading edge erosion can appear on the blade's leading edge in as early as two to three years into operation $[17,18]$ and without timely treatment, delamination of the underlying composite layers can also occur. Repairing the eroded blades can cost around $\$ 75,000$ [18]. The cost to replace the blades that are beyond repair would be much higher as a new blade costs about $\$ 200,000$ on average [19]. Although leading edge protection tapes are a popular solution, experiments have shown that they can also degrade the aerodynamic performance and cause up to $20 \%$ decrease in the maximum lift-to-drag ratio depending on the number of tape layers and the Reynolds number as well as the airfoil geometry [20]. In addition, in cases where high winds and access difficulties delay or limit the maintenance process (e.g. offshore wind turbines) the deterioration process may be accelerated leading to severe damage and longer downtimes [21].

This paper is the second part in a two-part paper that presents a multi-disciplinary computational framework for rain erosion prediction in wind turbine blades. Using the rainfall history at the wind turbine location, the blade shell structure and mechanical properties and the operational conditions of the wind turbine, the desired output of the framework is the incubation period or the time it takes for the blade coating to develop undesirable roughness. In part I [22], we developed a compound stochastic point process model of rain micro-structure that is capable of generating realistic three dimensional fields of raindrops for any rain intensity. In addition, we developed a multi-resolution CFD simulation strategy and an interpolation scheme that allows for efficient, yet accurate, calculation of raindrop impact pressure profile evolution for raindrops of various sizes in a given rain scenario. In part II, we integrate the ingredients developed in part I with new ingredients, i.e., high fidelity finite element models for transient stress analysis, fatigue damage calculations and probabilistic fatigue life estimations. We begin with importing the predicted impact pressure profiles of raindrops in realistic rain scenarios into high-fidelity finite element models of the blade shell structure to perform transient stress analysis and to obtain the cyclic stresses induced in the coating layer. This is followed by a fatigue analysis step which employs a cumulative damage rule to calculate the fatigue damage pattern of individual 
raindrop impacts. Using the intensity-specific three dimensional fields of raindrops, these individual damages are then superimposed and fatigue damage accumulation rates are calculated for any given rain intensity. The expected fatigue life of the blade coating is finally estimated by combining these accumulation rates and the statistics of the rainfall intensity at the turbine's location.

\section{Transient stress analysis in the blade shell}

The region of interest in the erosion study is the tip of the blade which is most frequently subjected to the highest impact velocities. Using impact pressure predictions available for all the drops in a given rain scenario (see Figure 1, see also [22]), the rain-induced stresses in the blade shell can be calculated using a detailed transient stress analysis. The wind turbine blade shell is usually manufactured by stacking several layers of composite and homogeneous materials. Due to the proprietary nature of the shell structure of the blades, there is not much detailed data available for the layup schedule and material properties. Researchers at Sandia National Laboratory have carried out a scaling study for a 100-meter-blade wind turbine (SNL100-00) in which they have designed a composite layup for the upscaled blade [23]. This structure is shown in Figure 2 for the blade's tip region. As mentioned earlier, wind turbines with blade lengths in this range are the most susceptible to rain erosion. Therefore, while there exists more detailed blade shell layup schedules in the literature (see [24]), we use the SNL100-00 blade shell structure specifications in this study. The mechanical properties for the materials used in this structure are listed in Table 1.

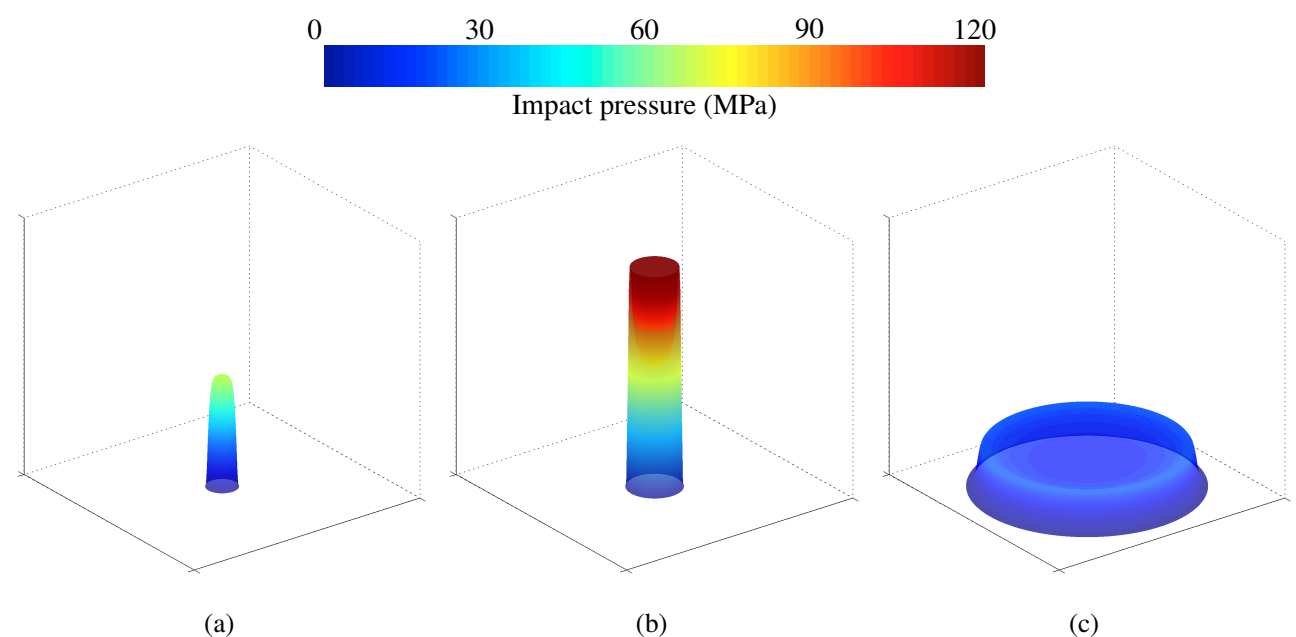

Figure 1: Evolution of the impact pressure profile for a $1 \mathrm{~mm}$ drop colliding with the surface at $100 \mathrm{~m} / \mathrm{s}:$ (a) $\mathrm{t}=30 \mathrm{~ns}$ (central peak), (b) $\mathrm{t}=70$ ns (lateral peak), (c) $\mathrm{t}=900$ ns; see [22]

\subsection{Finite element model}

ABAQUS/Explicit ${ }^{\circledR}[25]$ finite element software is used for modeling the composite layup and transient stress analysis. The model is shown in Figure 3a. Taking advantage of the symmetry of normal drop impact, only a quarter 
Table 1: Mechanical properties for the SNL100-00 blade layup.

\begin{tabular}{lllll}
\hline Material & $\begin{array}{l}E_{1} \\
(G P a)\end{array}$ & $\begin{array}{l}E_{2} \\
(G P a)\end{array}$ & $v_{12}$ & $\begin{array}{l}G_{12} \\
(G P a)\end{array}$ \\
\hline Gelcoat & $\mathbf{3 . 4 4}$ & $\mathbf{3 . 4 4}$ & $\mathbf{0 . 3}$ & $\mathbf{1 . 3 2}$ \\
Foam & 0.256 & 0.256 & 0.3 & 0.098 \\
SNL Triax & 27.7 & 13.65 & 0.39 & 11.8 \\
\hline
\end{tabular}

of the plate was modeled. The symmetry faces were assigned appropriate translational $(U)$ and rotational $(R)$ boundary conditions, i.e. $U_{x}=R_{y}=R_{z}=0$ for the face normal to the $x$ axis and $U_{y}=R_{x}=R_{z}=0$ for the face normal to the $y$ axis. The subscripts indicate the direction of translation or the axis of rotation. The external side faces were padded with semi-infinite elements (CIN3D8 in ABAQUS nomenclature) to prevent any undesirable stress wave reflection $[26,27,28]$. This step is taken since the plate is considered to be a small patch from a very large medium. The bottom face of the plate is fixed as this is a common approach for particle impact simulations $[29,30,31,26,27,32]$. The layers in the blade shell structure are assumed to be perfectly bonded. Three-dimensional 8-node brick elements with reduced integration (CR3D8 in ABAQUS nomenclature) are used to mesh the Gelcoat and foam layers, while the triaxial composite layers are modeled with 4-node shell elements (S4R in ABAQUS nomenclature).

\begin{tabular}{|c|c|}
\hline Gelcoat & $0.6 \mathrm{~mm}$ \\
\hline SNL Triax & $5 \mathrm{~mm}$ \\
\hline Foam & $5 \mathrm{~mm}$ \\
\hline SNL Triax & $5 \mathrm{~mm}$ \\
\hline
\end{tabular}

Figure 2: Blade shell layup near the blade's tip for the SNL100-00 100-meter-blade wind turbine [23].

The stresses in the Gelcoat layer were calculated for two separate cases: 1) using the entire layup structure and 2) using only the Gelcoat layer with a rigid bottom face. It was observed that while the inclusion of the composite structure in the model increases the computational cost dramatically, it does not alter the stresses within the Gelcoat layer significantly. In fact, the peak stresses in the Gelcoat layer in the two cases differ only by less than 5\%. This, together with the fact that the scope of the present study is limited to the time period before surface roughening starts to appear (i.e. the incubation period), allows for simplifying the finite element model to one that involves a patch of the Gelcoat layer padded with semi-infinite elements. This simplification reduces the material properties to a single isotropic material property. In addition, since the impact pressure load is normal to the surface, an axisymmetric model, shown in Figure 3b, can be used. The appropriate boundary conditions for an axisymmetric analysis are applied to the model, i.e. $U_{x}=0$ on the axis of symmetry. The bottom face of the plate is fixed $U_{x}=U_{z}=0$. The external face parallel to the axis of symmetry is padded with semi-infinite elements (CINAX4 in ABAQUS nomenclature). 
Axisymmetric 4-node elements with reduced integration (CAX4R in ABAQUS nomenclature) are used to mesh the Gelcoat.

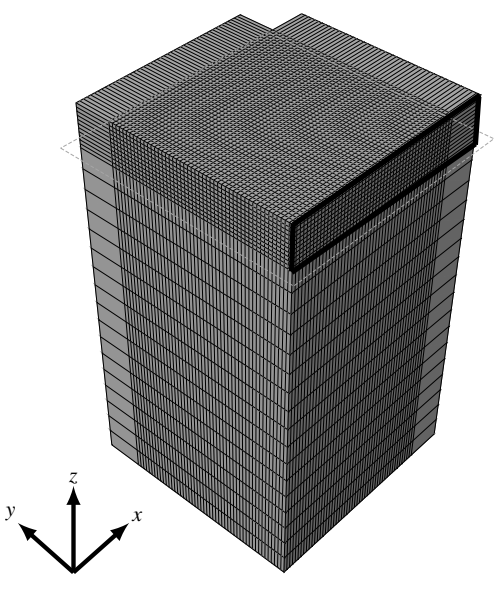

(a) 3D Multi-layered model of the layup

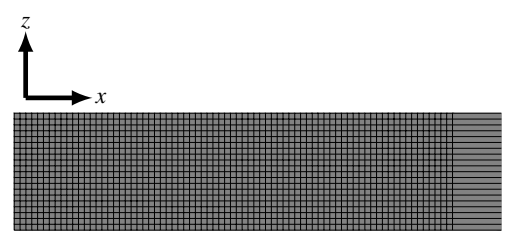

(b) Axisymmetric model of the Gelcoat layer representing the marked face in Figure 3a.

Figure 3: The three dimensional and axisymmetric models created in ABAQUS.

\subsection{Convergence and geometrical considerations}

Finite element simulations are performed separately for a large family of raindrops ranging from 0.1 to $7 \mathrm{~mm}$ in diameter. In each simulation, the plate size is chosen based on the raindrop size. The plate size is chosen large enough such that the high stress regions are captured. Points far from the impact point have negligible stresses compared to the overall maximum values as shown in Figure 5.

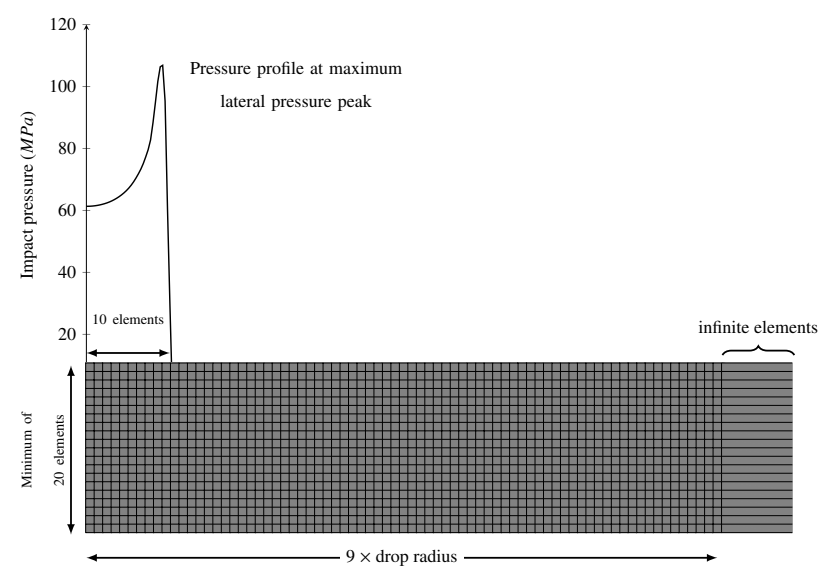

Figure 4: The relation between the finite element mesh resolution and the impact pressure length scale.

In addition, the finite element mesh size is chosen based on the drop size. This is done to include small features of the impact pressure profile in the transient stress analysis such as those near the lateral peak. The finite element mesh 
is fine enough to ensure that the pressure profile covers a radius of ten elements at the time of lateral peak maximum. This is illustrated in Figure 4. Further refinement of the mesh does not change the overall maximum stress in the plate.

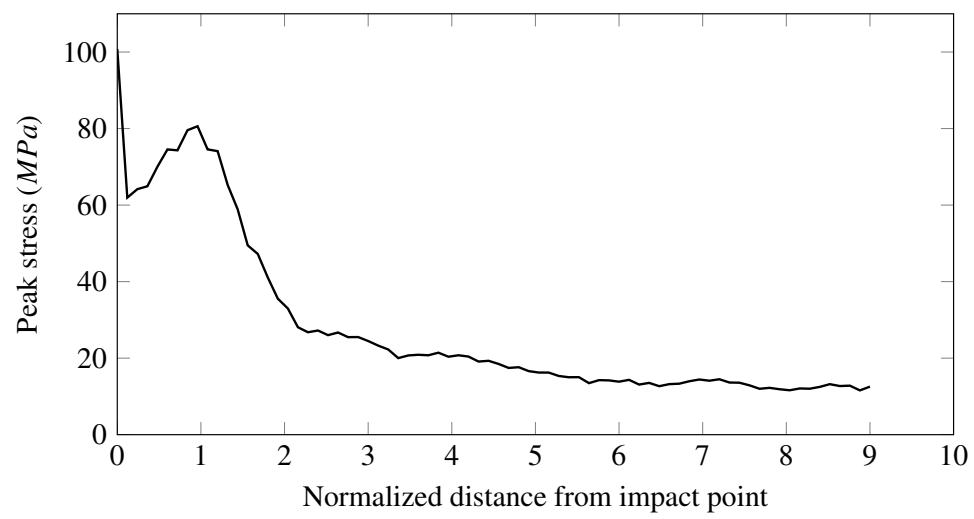

Figure 5: Peak stress in the coating layer vs. the distance from the impact point for a $2.25 \mathrm{~mm}$ drop impact at $100 \mathrm{~m} / \mathrm{s}$.

Prior to the fatigue analysis, a valid question is whether the stresses from different impacts interfere with each other. Fortunately, the time lag between consecutive raindrop impacts is much (almost three orders of magnitude) larger than the time needed for the stress waves resulting from a single drop impact to disappear. Therefore, it is safe to assume that the stress waves from different impacts do not interact.

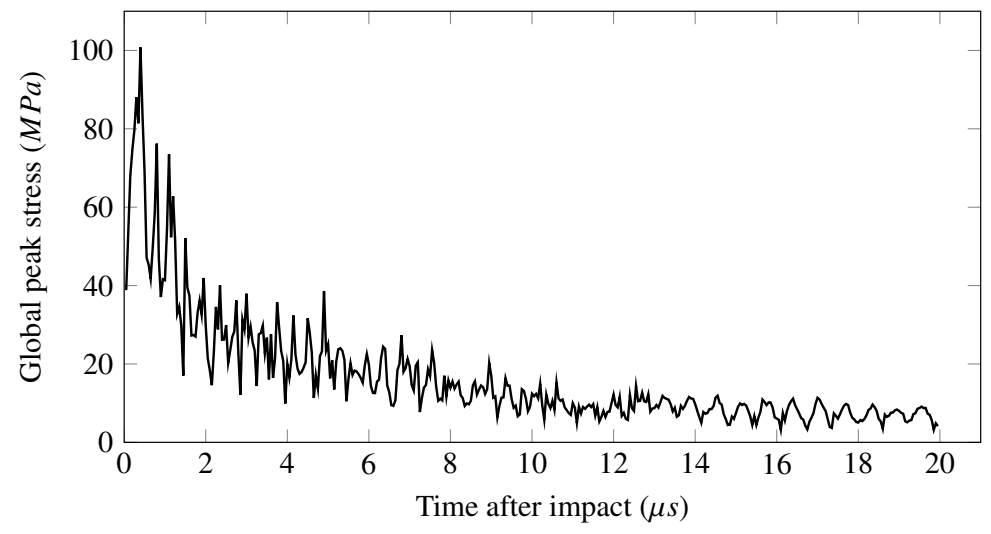

Figure 6: Global peak stress in the coating layer vs. time for a $2.25 \mathrm{~mm}$ drop impact at $100 \mathrm{~m} / \mathrm{s}$.

\subsection{Finite element analysis automation via ABAQUS scripting environment}

ABAQUS scripting interface is used to perform the stress analysis for all raindrops. Each impact is simulated separately on a plate with the dimensions and mesh resolution conforming to the drop size. The script takes several input parameters such as the material properties and raindrop size. Based on this information, the pressure profile of the desired drop size is interpolated and the plate model is automatically created, meshed and analyzed. The interpolated pressure profiles for all frames (in time) are written to separate files. During each analysis, raindrop pressure loads are imported using the VDLOAD user subroutine. ABAQUS applies the pressure load to the integration 
points on the exposed face of the surface elements. The coordinates of these integration points are made available during every call to the VDLOAD subroutine. The subroutine is programmed to access the pressure profile of the appropriate frame based on the elapsed time in the analysis. It uses the coordinates of each integration point and an inverse distance interpolation to extract the applied pressure from the pressure profile.

As mentioned earlier, the goal of performing the stress analysis is to obtain stress-time histories for all points within the coating layer. In the section that follows, these stress-time histories will be used to calculate the fatigue damage pattern associated with individual raindrops and the expected fatigue lifetime of blade coating. In order to correlate the fatigue damage with raindrop size, a range of raindrop sizes from 0.1 to $7 \mathrm{~mm}$ at $0.1 \mathrm{~mm}$ intervals are selected. This choice is based on the range of raindrop sizes that could exist in a typical realization of the rain texture model.

\section{Fatigue analysis framework}

The process of rain-induced erosion can be considered as a case of fatigue damage initiation and evolution as it involves repeated low-stress loadings that cause failure over an extended time period [33, 34, 35, 36, 37, 38, 39, 40, 41]. Fatigue properties of materials are usually expressed in the form of S-N curves, i.e. stress amplitude versus the number of cycles to failure. Similarly, liquid impact erosion behavior of materials has been presented as erosion endurance curves, i.e. the impact velocity versus the number of impacts either to complete the incubation period or to increase the erosion depth by a certain amount. Striking similarities have been found between fatigue S-N curves and erosion endurance curves examples of which are shown in Figure 7 [42]. In fact, it has been reported that the completion of the incubation period in erosion coincides with macroscopic fatigue failure [43]. In addition, it was mentioned earlier that the maximum impact pressure rapidly grows as it moves from center to the periphery of the contact area. Such sharp changes in loading are believed to have an adverse effect on fatigue performance [34, 42]. This further justifies the use of fatigue analysis for the prediction of the incubation time.

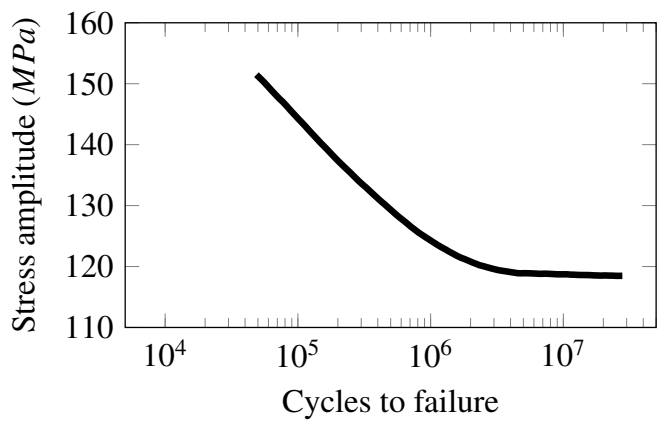

(a)

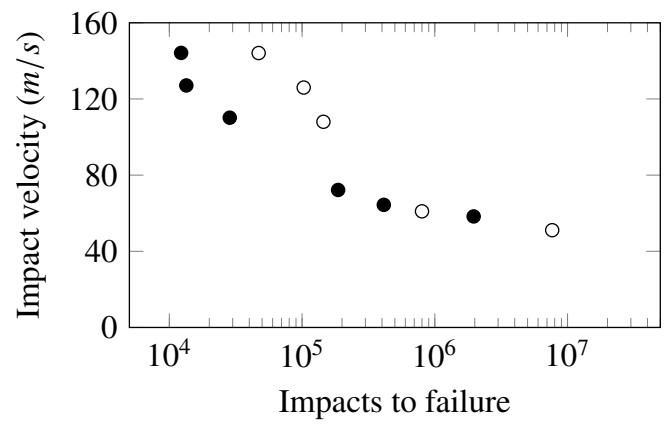

(b)

Figure 7: Comparison of fatigue S-N curve (a) and erosion endurance curve (b) for mild steel. The solid circles indicate the completion of the incubation period and the open circles indicate the erosion depth increase by $100 \mu \mathrm{m}$ [42]. 
The homogeneous Gelcoat layer is the region where the highest stresses are observed. Above all, fatigue essentially starts from the surface. Upon the completion of the incubation period, surface roughness develops which reduces the aerodynamic performance in the case of the wind turbine blade. Therefore, it is sensible to focus on the fatigue life prediction of the outermost layer of the shell structure, i.e. the Gelcoat layer. Furthermore, the finite element analysis results indicate that, as expected, the stresses within the coating layer remain well within the elastic limit. Therefore, failure is expected to occur at a high number of cycles suggesting that a stress-life approach appropriate for high-cycle fatigue should be adopted.

\subsection{Fatigue life prediction through cumulative damage index}

From a practical point of view, there are three categories of fatigue life prediction techniques for constant or variable amplitude stress loadings: micro-mechanical models, empirical models and cumulative damage models [5]. Micro-mechanical models are still under exploration and have so far been limited to single failure modes. Empirical models commonly use stiffness or strength degradation during fatigue loading. However, these models require comprehensive experimental data and are very sensitive to the material structure. On the other hand, cumulative damage models are extensively used for fatigue life predictions owing to their relative simplicity. The most popular of these methods is the Palmgren-Miner sum index [44], which defines a damage parameter that increases linearly over time. The damage parameter is given by

$D=\sum \frac{n_{a}}{N_{a f}}$

where $n_{a}$ and $N_{a f}$ are respectively the number of cycles applied and the number of cycles to failure at stress amplitude $a$. The summation is carried out over all stress amplitude values that are present in a stress-time history. Fatigue failure occurs when the damage index equals one. Note that, under the Miner's rule assumption, the damage index is not a physical parameter and is not necessarily related to the stiffness or strength of the material. Also, the order of loadings does not matter, i.e. the damage of any cycle is independent of the damage of the previous cycles [5]. It is also common to represent the damage in an expected value sense, which makes damage comparisons more general and meaningful [44]. Miner's rule alongside Rainflow cycle counting and mean stress correction (see [44, 45]) has been used previously for fatigue analysis in wind turbines [23, 41, 46, 47]. The idea is that a point of interest has reached its fatigue life when its associated damage index is equal to 1 . In order to find the fatigue life, the linearity of damage accumulation is invoked, i.e.

$t_{f}=\frac{t_{s}}{D_{s}}$

where $t_{f}$ is the fatigue life, $t_{s}$ is the duration of the loading (e.g. rain duration) and $D_{s}$ is the damage accumulated over time $t_{s}$. 


\subsection{Multiaxial fatigue}

Uniaxial state of stress is the simplest case in which a single stress value can be compared directly to the stress values on the S-N curve. However, when more than one of the stress components are nonzero, a multiaxial state of stress is present in which case direct application of the S-N curve is not possible. In such cases, equivalent stress methods are commonly used. They serve to reduce the stress components into a single equivalent stress which will be compared with the stress values on the S-N curve. Signed Von Mises stress, signed Tresca stress and the absolute maximum principal stress (AMPS) are examples of such equivalent stresses [44]. The "absolute maximum" value in AMPS indicates that the sign of the principal stress is kept. Von Mises and Tresca stresses are always positive. This undesirably affects the alternating nature of the stress-time history. Therefore, the largest principle stress (in an absolute value sense) is used to determine the sign of the stress signal when Von Mises or Tresca stresses are used. In this study, we use the absolute maximum principal stress to handle the multiaxial state of stress in our fatigue damage calculations. It should, however, be noted that other damage index definitions (see e.g. [48]) can be used to calculate damage from individual stress components. In fact, such definitions coupled with constant life diagrams have been used for probabilistic estimation of fatigue life in composite wind turbine blades under non-proportional loading conditions [24].

\subsection{Cycle counting and nonzero mean stress correction}

S-N curves are often obtained from fatigue tests that are performed under a controlled uniaxial stress state and a regularly varying stress amplitude. Stress-time histories in real-world applications rarely have such regularity. Nevertheless, in order to use Equation (1), the number of cycles at every stress amplitude and mean stress has to be known. Given a stress-time history, various cycle counting methods can provide this information. These include Rainflow counting, level crossing, range-mean and range counting. Among these methods, Rainflow cycle counting is used most often in fatigue analysis. The most common procedure for Rainflow counting involves the following steps [44]: First, only the peaks and valleys of the stress signal are extracted and the rest of the points are ignored. Second, the start and end of the signal are forced to have the same value by copying the starting point to the end. Third, the highest peak is found and the signal is reordered like a ring such that this peak is both the beginning and the end of the signal. Fourth, starting from the beginning, consecutive sets of 4 points are chosen and the following rules are applied. If the middle segment is shorter than the first and third, then it is extracted as a Rainflow cycle as shown in Figure 8. If a cycle is not found, the next 4 points are checked. When a cycle is found, the search starts from the beginning of the signal. It should be noted that, similar to the Palmgren-Miner rule, Rainflow method also assumes that the loads are independent and that the order of loading is insignificant. More details on the Rainflow counting method is given in $[5,44,45]$. The output of Rainflow counting, i.e. the number of cycles at all stress amplitudes and mean stresses is best represented by the Rainflow matrix such as shown in Figure 9. 


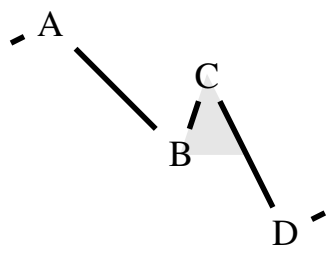

(a)

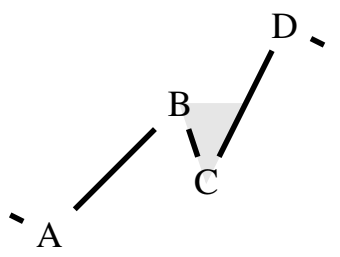

(b)

Figure 8: Rainflow cycles [44]. In cases (a) and (b), the segment BC is removed and a cycle is counted.

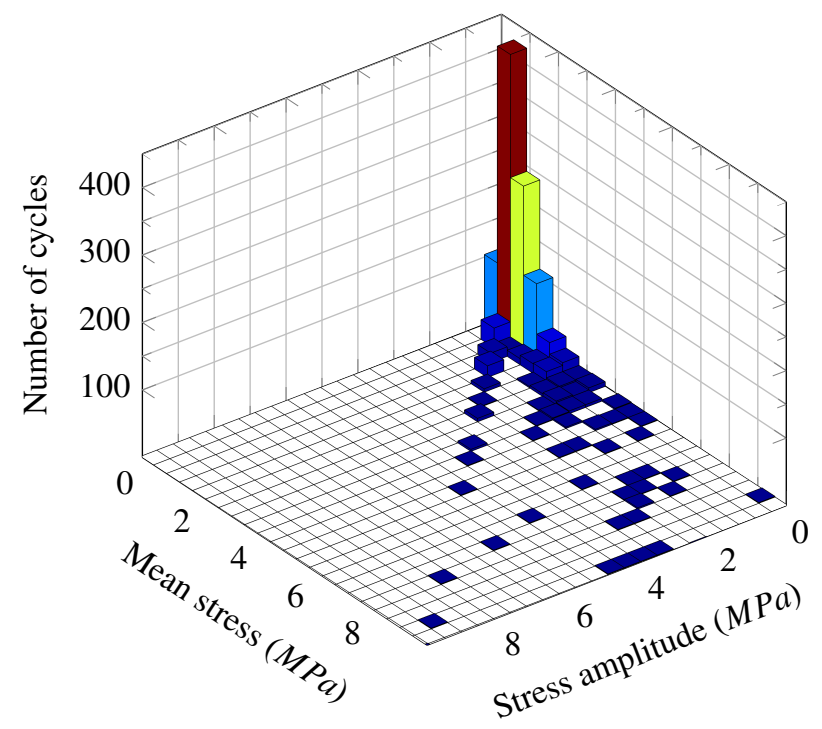

Figure 9: Rainflow matrix for an arbitrary point in the reference plate which shows the number of cycles at every possible stress amplitude and mean stress.

The other important thing to note is that the fatigue tests are usually performed with a zero mean stress. The mean stress is defined as the average of the maximum and minimum stresses in a stress-time history. However, as shown in Figure 9, nonzero mean stresses are present in the signal. To account for nonzero mean stresses, many mean stress correction equations have been proposed. Among these, the linear Goodman relation [5] is one of the most commonly used. The Goodman equation corrects the amplitudes of the Rainflow counted signal according to their mean stress using the following relationship

$\sigma_{a}^{\prime}=\frac{\sigma_{a} U T S}{U T S-\sigma_{m}}$

where $\sigma_{a}^{\prime}$ is the corrected amplitude, $\sigma_{a}$ is the original stress amplitude, $\sigma_{m}$ is the mean stress and UTS is the ultimate tensile strength. Note that UTS in Equation (3) should be substituted with the ultimate compressive strength $U C S$ for negative mean stresses. 


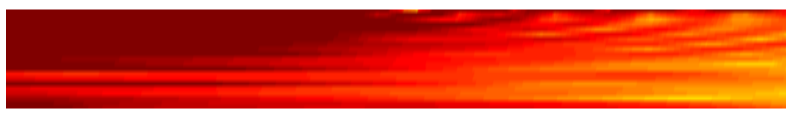

$0.1 \mathrm{~mm}$

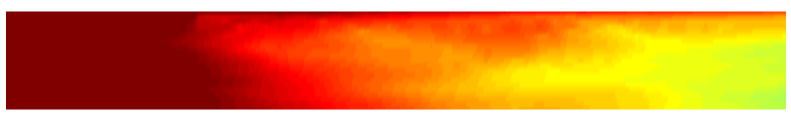

$0.4 \mathrm{~mm}$

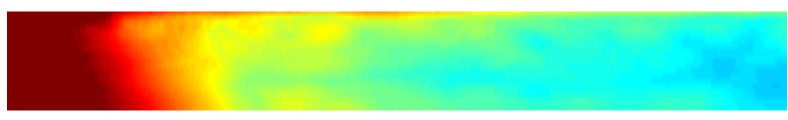

$1.1 \mathrm{~mm}$

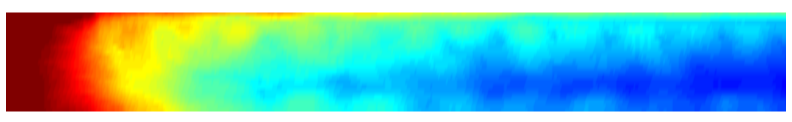

$1.5 \mathrm{~mm}$

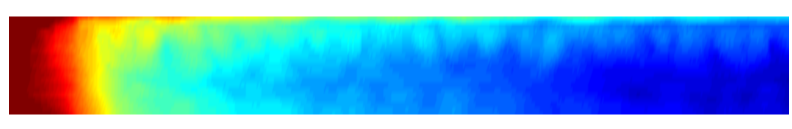

$2.5 \mathrm{~mm}$

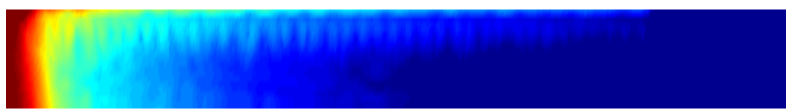

$5.5 \mathrm{~mm}$

$7.0 \mathrm{~mm}$
$0.3 \mathrm{~mm}$

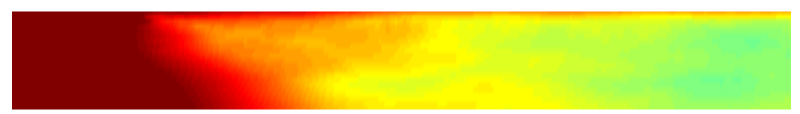

$0.7 \mathrm{~mm}$

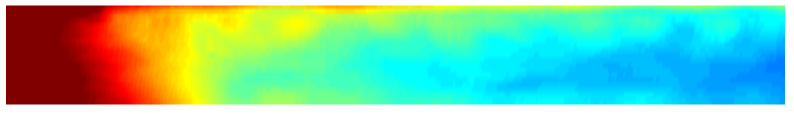

$1.2 \mathrm{~mm}$

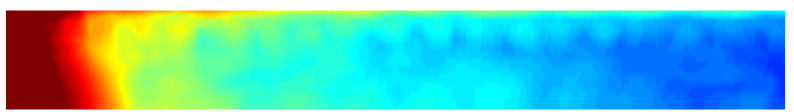

$2.0 \mathrm{~mm}$

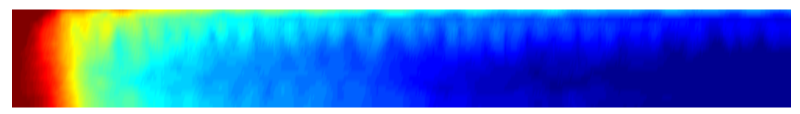

$3.7 \mathrm{~mm}$

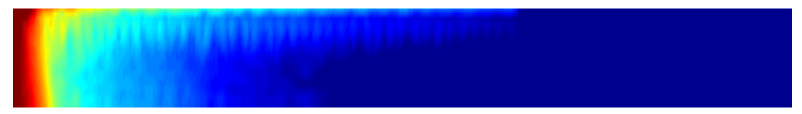

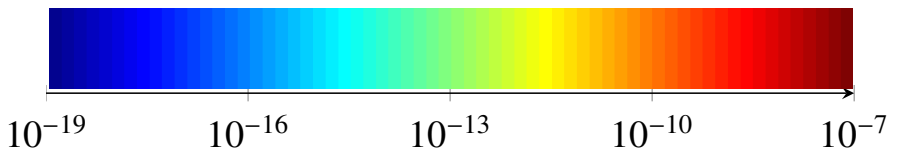

Figure 10: Fatigue damage pattern for raindrops of various diameters on normalized axes shown as $\log ($ damage $)$. The $\mathrm{x}$ axes stretch from impact point to $8 \times$ drop radius.

\section{Estimation of expected coating fatigue life}

\subsection{Single drop impact-induced damage}

It was mentioned earlier that the timescales associated with raindrop impact-induced stresses and the lag between individual raindrop impacts in a typical rain scenario are significantly different. Following the procedure described in the preceding sections, the fatigue damage pattern (spatial variation of damage index) in the coating layer is obtained for a representative family of raindrops $(0.1$ to $7 \mathrm{~mm})$. This allows for breaking down the damage index at a given point to contributions of individual raindrops. These fatigue damage patterns will then be used through an interpolation scheme to obtain the damage pattern for any raindrop size. In order to facilitate the interpolation, the coating domain dimensions are normalized by the drop radius in the $x$ (radial) direction and by the coating thickness in the $z$ (vertical) direction. These normalized damage patterns are then mapped to a common grid. This way, the damage patterns of all drop sizes can be compared and interpolation based on drop size can be performed in a more straightforward manner. 
Figure 10 depicts the damage patterns in the normalized domain for several drop sizes, where it is seen that the damage is normally at its maximum close to the surface, while Figure 11 shows how the damage indices for different points in the normalized domain vary as a function of drop diameter. Once the normalized damage pattern for a given drop size is calculated, it should be expanded to reflect the actual size of the drop. It should, however, be noted that the results depicted in these figures pertain to a particular Gelcoat thickness and material properties and as such will change as the characteristics of Gelcoat layer change relative to geometrical and material properties of its underlying layer. In addition, the details of the FE model for a given Gelcoat thickness and layup schedule may affect the availability of stress data through thickness and consequently the value of fatigue damage indices (see [49]).

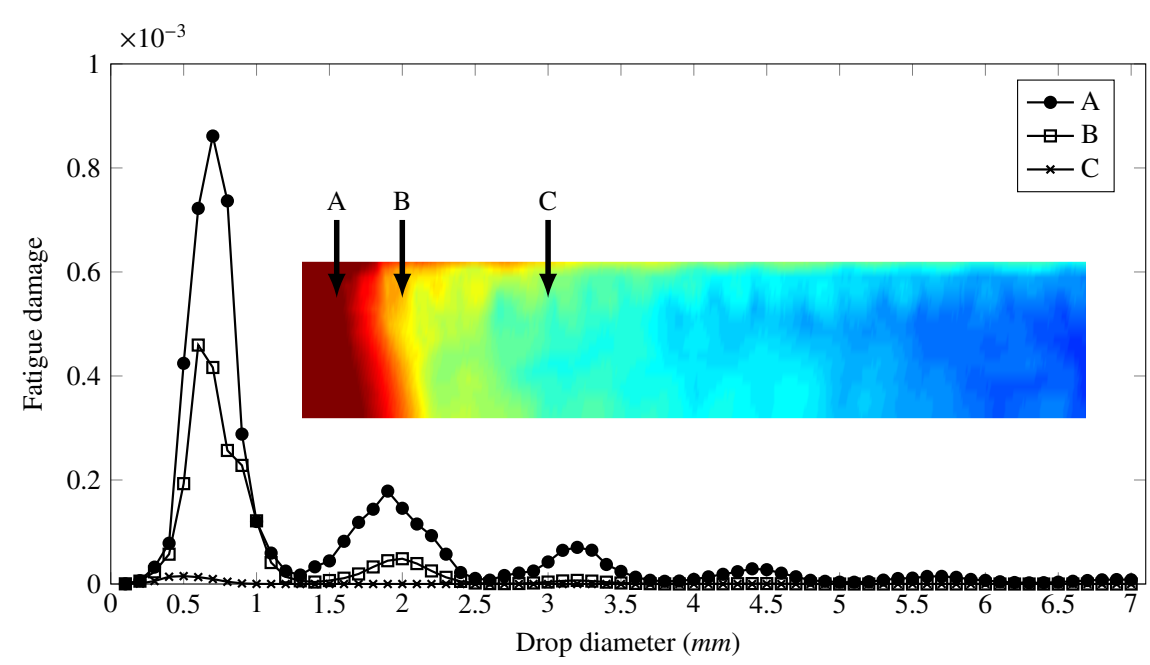

Figure 11: Damage index vs. drop diameter for different points in the normalized domain.

\subsection{Damage accumulation rate-rain intensity relationship}

Having obtained the fatigue damage pattern for any drop size, the next step in fatigue life calculation is to correlate the rate of fatigue damage accumulation to the rain intensities. These rain intensities come from the statistical analysis of rainfall history (see part I [22]). In order to calculate the fatigue damage accumulation rate, the individual raindrop fatigue damages are superposed on a small target plate. This superposition is done according to the 3D raindrop field for a given rain intensity generated from the stochastic rain texture model developed in part I [22]. It should be emphasized at this point that the main goal is to predict a single fatigue life estimate and not to present a fatigue life map across the surface. After all, the spatial distribution of drops is uniform and over an extended time period uniform erosion within the small surface patch under consideration is expected. Nevertheless, in order to calculate the fatigue damage for a typical point on the surface of the target blade, all the raindrops that contribute to fatigue at this point need to be considered. We note that the largest raindrop creates the highest stresses in the plate as it has the highest kinetic energy at the time of impact. The stress waves dissipate after they travel a certain distance from the point of impact. Denoting this distance by $r$, in order to calculate the fatigue damage at a point such as A in Figure 12, one would only need to consider the drop impacts that collide with the surface within a radius of $r$ from A. This, 
loosely speaking, is equivalent to considering all the impacts within a square area circumscribing a circle of radius $r$ centered at A. If the fatigue life prediction for more points is desired, e.g. the points in a small circular area of radius $d r$ centered at $\mathrm{A}$, then the square area should at least contain a circle of radius $r+d r$.

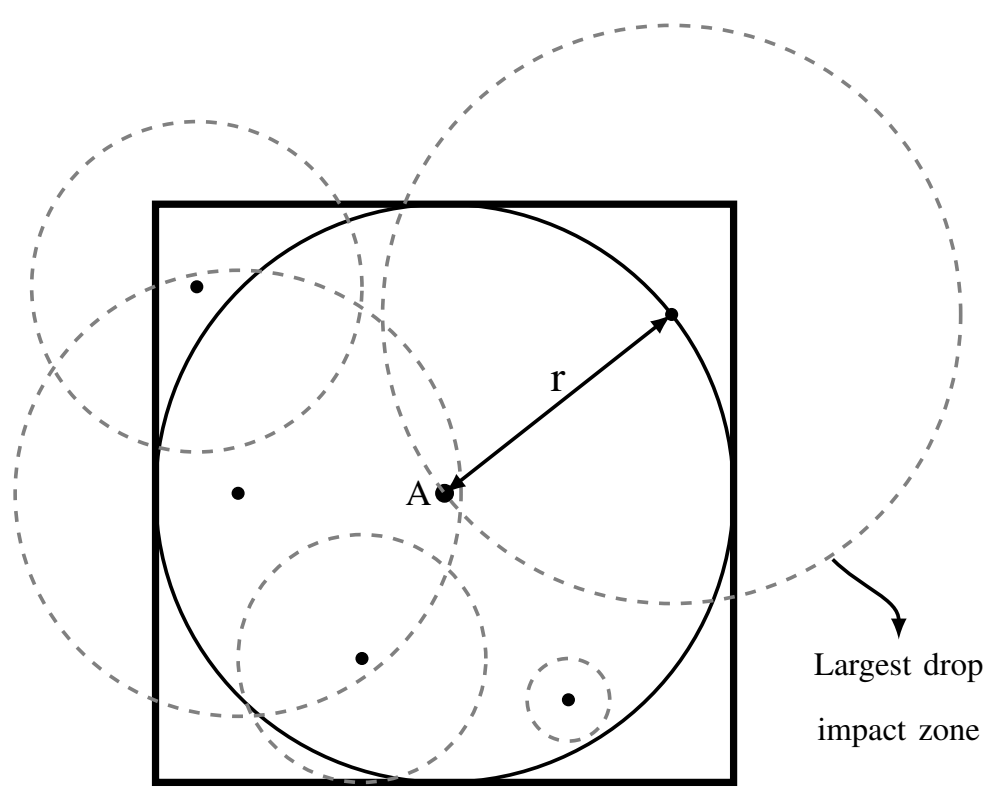

Figure 12: Determination of the "influence" area from the size of the area affected by the impact of the largest raindrop.

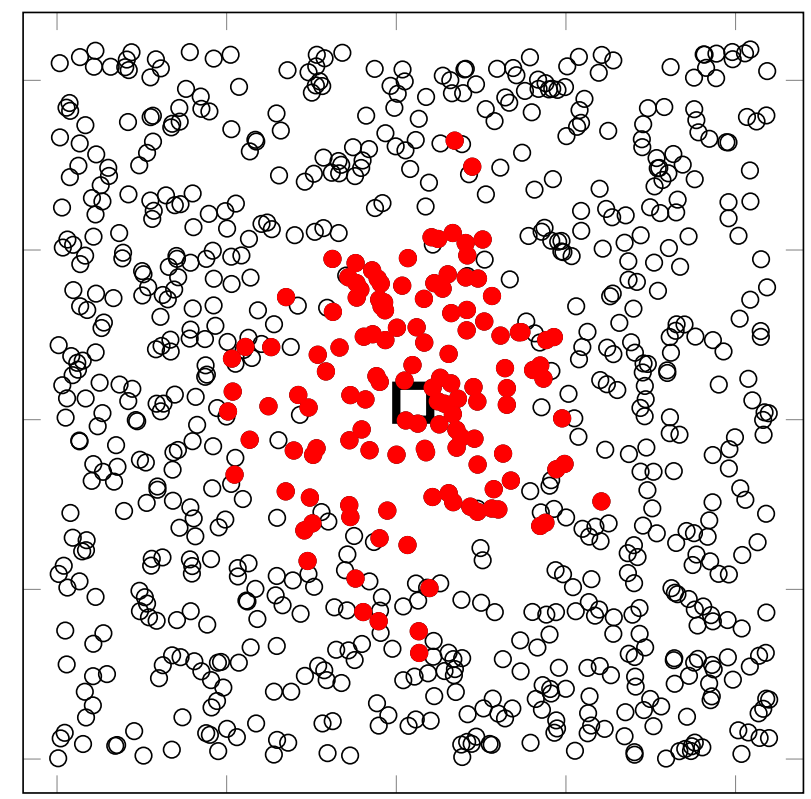

Figure 13: Effective raindrop impact points in a $2.1 \mathrm{~cm} \times 2.1 \mathrm{~cm}$ domain. The $1 \mathrm{~mm} \times 1 \mathrm{~mm}$ zone is shown as a square in the center. The red dots show the drops that are causing fatigue damage in this zone. The rain intensity is 0.25 $\mathrm{mm} / \mathrm{hr}$ and the duration is 1000 seconds. 
Now, let us consider a small area, say $1 \mathrm{~mm} \times 1 \mathrm{~mm}$, on the surface with which the raindrops are colliding. This area is discretized to facilitate the superposition of raindrop impact-induced damages. From the 3D field of raindrops, all the raindrops that cause fatigue damage on this area have to be considered. This includes some raindrops that are impacting the surface outside this area as schematically depicted in Figure 13. The large square represents the base of the raindrop column. The small square at the center is the zone for which the fatigue life is calculated. Raindrop impacts that contribute to damage in this zone are depicted by solid circles. The sizes of raindrops are not shown.

Another consideration in generating the raindrop field is the rain duration and its relation to damage accumulation rate. To address this issue, we note that the damage accumulation rate needs to be calculated in an average sense. This is because, according to the rain texture model, the raindrops are uniformly distributed in space. Therefore, in the long run, what an arbitrary point in the target area collects in terms of fatigue damage is independent of its location. In light of this argument, the accumulated fatigue damage is recorded over time by taking the average of the damages of all points in the discretized target area. Tracking this average damage over time yields a graph such as the one shown in Figure 14. The fatigue damage accumulation rate $\alpha$ is then the slope of the linear fit to these points which is shown as the solid straight line in this figure. An important note here is that the accumulation rate calculated in this way will be a function of rain duration for short rain durations. However, as the rain duration increases, $\alpha$ converges to a constant value. This behavior is depicted in Figure 15. The rain duration after which the value of $\alpha$ remains constant is chosen to be the rain duration that can be used to calculate the damage accumulation rate.

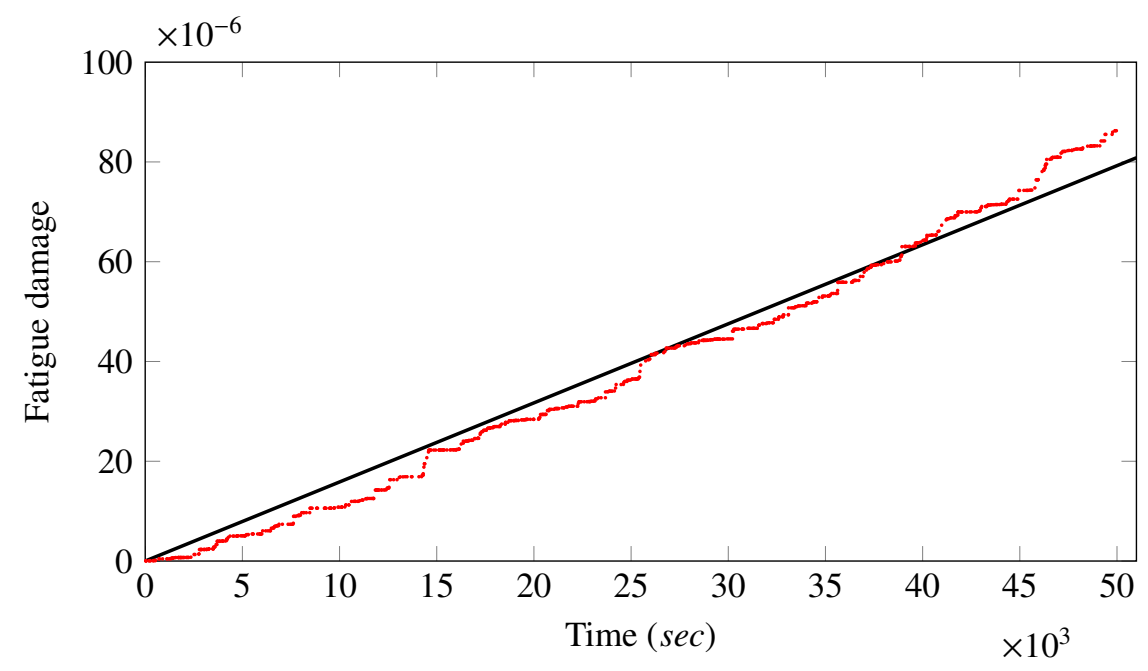

Figure 14: Accumulation of the average fatigue damage over time. The black line shows the fit of the form $y=\alpha x$. 


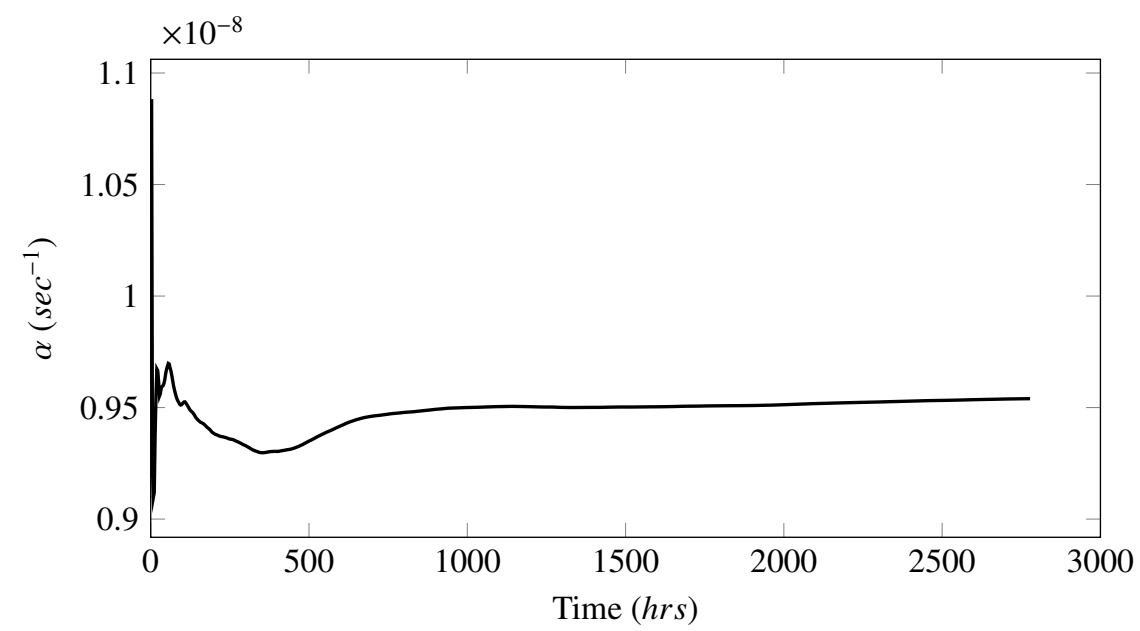

Figure 15: Convergence of the calculated fatigue damage accumulation rate $\alpha$ vs. rain duration for $1 \mathrm{~mm} / \mathrm{hr}$ rain.

It was mentioned earlier that the fatigue damage at a given point in the target area is obtained by superposing the damages associated with individual raindrop impacts in a rain scenario. In order to facilitate the superposition process, the target area needs be discretized and the damage values on the grid points need to be calculated via interpolation. Therefore, it is necessary to show that the calculated accumulation rate $\alpha$ is independent of the target area grid resolution. This convergence behavior is shown in Figure 16 where it is seen that $\alpha$ converges at 20 grid points per thickness.

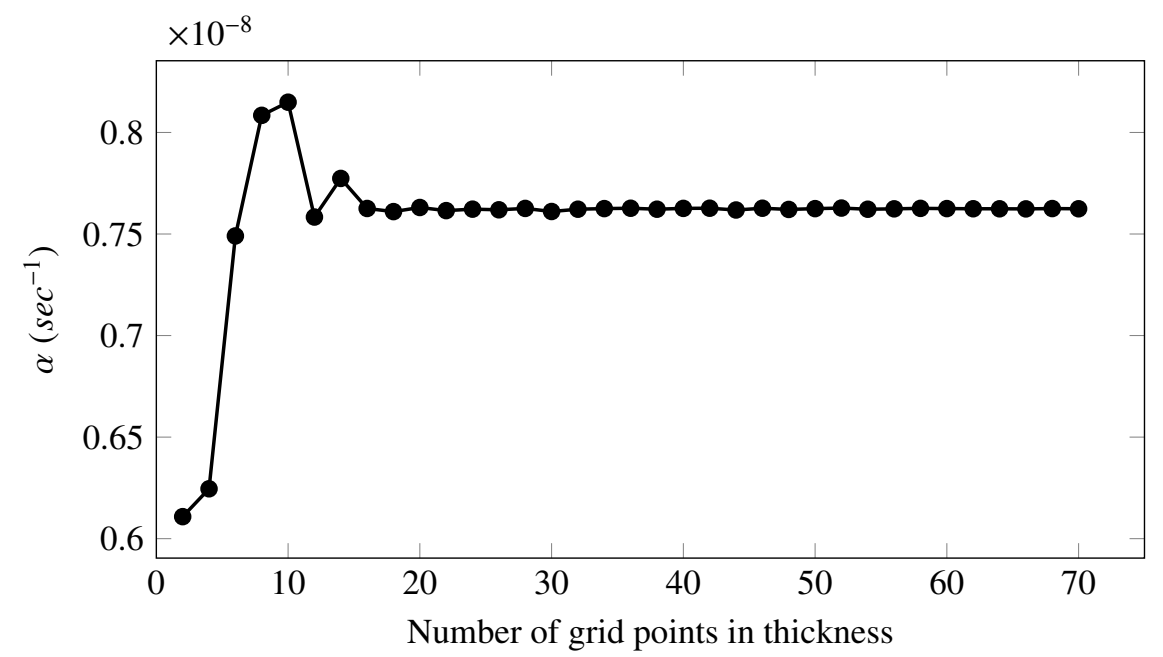

Figure 16: Convergence of the calculated fatigue damage accumulation rate $\alpha$ vs. the target plate grid resolution. The rain intensity and duration are $1 \mathrm{~mm} / \mathrm{hr}$ and 10000 seconds respectively. 


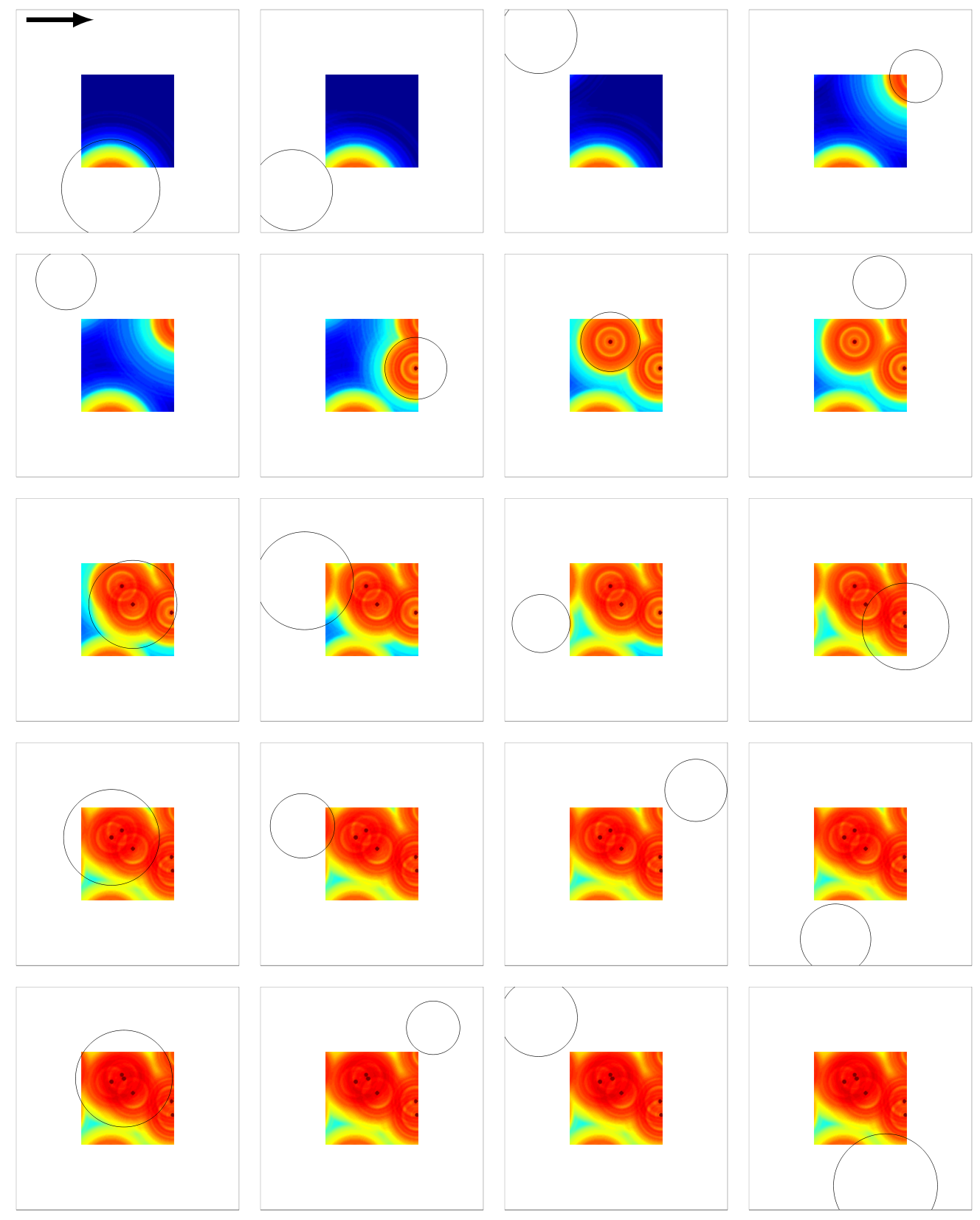

Figure 17: Fatigue damage superposition on the target plate from the impact of 20 consecutive raindrops. The black circles show the diameter of the raindrops. For visualization purposes, the impact zone is $2.9 \mathrm{~mm} \times 2.9 \mathrm{~mm}$ and the target plate at the center is $1.5 \mathrm{~mm} \times 1.5 \mathrm{~mm}$. The arrow on top left corner indicates the direction of increasing time.

With the appropriate rain duration and target area resolution determined, it is now possible to calculate the true fatigue damage accumulation rate $\alpha$ for any rain intensity. As mentioned earlier, this is achieved through the superposition of individual raindrop impact-induced fatigue damages. An example of this procedure is shown in Figure 17 for 20 consecutive impacts. The color contours provide a qualitative view of the fatigue damage for each impact and the open circles represent raindrop diameters. 
Following the procedure described above, the fatigue damage accumulation rate $\alpha$ on the outer surface of the coating is calculated for all rain intensities present in the rain intensity-duration histogram associated with the turbine's location (see part I [22]). The calculation of $\alpha$ is focused on the outer layer based on the assumption that fatigue essentially starts from the surface. In addition, the highest values of $\alpha$ are observed for the points on the surface. The results for all rain intensities are shown in Figure 18. It can be seen that, similar to the relationships between the average volume of water or average number of drops per unit volume of air and the rain intensity (see [22]), the fatigue damage accumulation rate $\alpha$ also has a power law relationship with rain intensity.

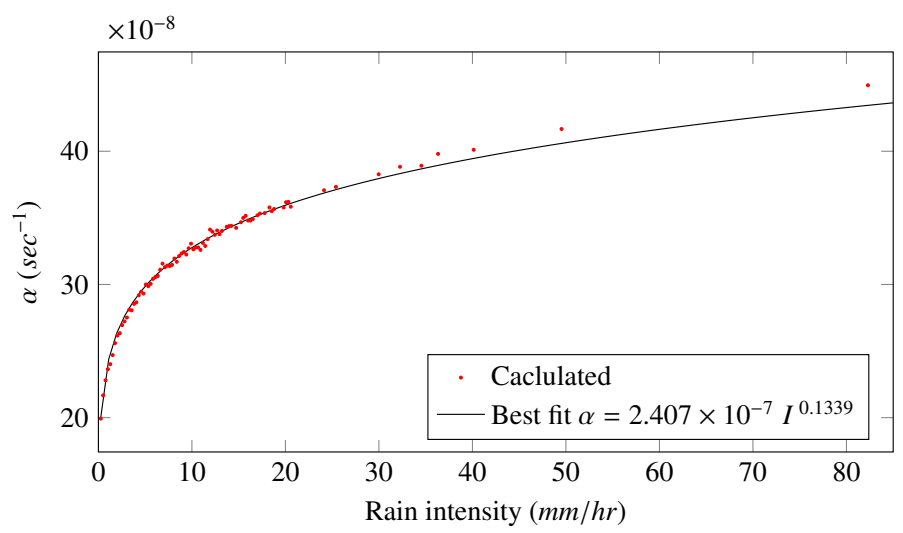

Figure 18: Calculated fatigue damage accumulation rate $\alpha$ vs. rain intensity.

\subsection{Expected fatigue life calculation}

Figure 19 shows how different ingredients of the proposed computational framework come together as a means to estimate the fatigue lifetime of the coating in composite wind turbine blades. Having established a relationship between the damage accumulation rate and the rain intensity, it is now possible to proceed to the final stage of fatigue life calculation. Such a calculation will be based on the expected amount of damage accumulated per year $\alpha_{\text {year }}$ for a given location which can be calculated using the following equation:

$\mathbb{E}\left[\alpha_{\text {year }}\right]=\sum_{I}\left(\alpha_{I} \frac{1}{\text { second }} \times t_{r} \frac{\text { hour }}{\text { year }} \times 3600 \frac{\text { second }}{\text { hour }}\right) P_{I}$

where $\mathbb{E}$ denotes expectation or average, $\alpha_{I}$ is the damage accumulation rate for the rain intensity $I$ (extracted from Figure 18), $t_{r}$ is the average total number of rain hours per year and $P_{I}$ is probability mass associated with rain intensity $I$ (see part I [22]). The expected fatigue life $t_{f}$, assuming that the coating reaches its fatigue life when the damage index is equal to 1 , can then be simply calculated from

$t_{f}=\frac{1}{\mathbb{E}\left[\alpha_{\text {year }}\right]}$

which results in a fatigue life of about 1.8 years in the case of the 100-meter-blade wind turbine (SNL100-00) installed in the city of New Bedford, MA. This estimate sounds reasonable when compared to field observations that indicate 
that wind turbine blades show signs of erosion between 2 to 3 years after installation. Having said that, we would like to emphasize that the goal of this work is not to present fatigue life numbers. Rather, the goal is to develop a multifaceted framework that can relate rain characteristics at a given location and turbine blade's structural and material properties as well as turbine's operational conditions to quantitative measures of the potential for rain-induced erosion at that location.

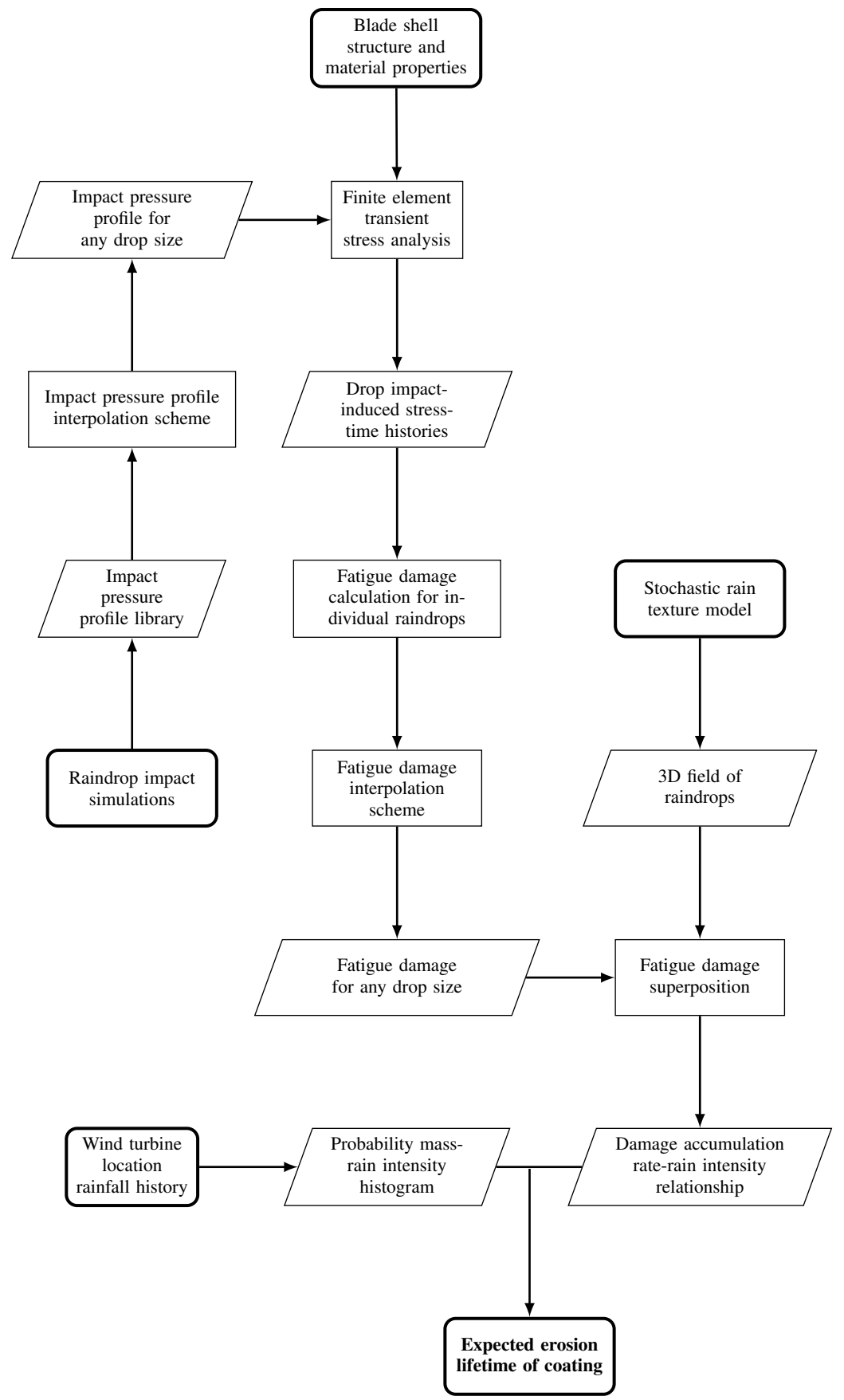

Figure 19: The ingredients of the proposed computational framework. 


\section{Concluding remarks}

With the growing interest in renewable energies in the past decade, the power output of wind turbines has increased significantly. Often manifested in the form of larger rotor blades, this increase has brought about new technological challenges. The large size of the blades results in very high blade tip velocities and an increase in the susceptibility of blades to rain erosion. This paper is the second part in a two-part paper that presents fundamental ingredients of a multifaceted computational framework to provide quantitative measures of rain erosion in wind turbine blades. Part I [22] involved realistic modeling of the rain texture through a compound stochastic point process and high fidelity, yet efficient, calculation of spatiotemporal variations of the impact pressure in the droplet-surface collision via multiresolution GPU-accelerated CFD simulations and an interpolation scheme. In part II we were concerned with high fidelity finite element analysis of transient stresses in the blade shell. Impact pressure profile predictions obtained in part I were imported into the finite element model and the stress-time histories within the blade coating were obtained. These stress-time histories were then fed into a multiaxial fatigue analysis framework based on cumulative damage rules to calculate the fatigue damage pattern for individual raindrops. These damage patterns were then integrated with intensity-specific three dimensional fields of raindrops, generated via the stochastic rain texture model developed in part I, to establish a relationship between damage accumulation rates and rain intensities. At the end, these accumulation rates, together with the statistics of rain fall history at the turbine location, provided a means for estimating the expected fatigue life of the blade coating as an indicator for the end of incubation period after which the wind turbine blade aerodynamic performance deteriorates due to the emergence of surface roughening.

\section{Acknowledgements}

This work was supported by a grant from the University of Massachusetts President's Office under the Science and Technology Initiative Program. This support is gratefully acknowledged. M. Raessi also gratefully acknowledges support from the National Science Foundation under the CBET Grant No. 1336232.

\section{References}

[1] U.S. Energy Information Administration, International Energy Outlook 2011, Tech. rep., U.S. Energy Information Administration (2011). URL http://www.eia.doe.gov/oiaf/ieo/index.html

[2] Global Wind Energy Council, Global Wind Report, Tech. rep. (2012).

[3] 4C Offshore, Vestas v164 wind turbine product specifications (2014). URL http://goo.gl/3vgT1U

[4] 4C Offshore, Samsung s7.0-171 wind turbine product specifications (2014). URL http://goo.gl/HRQfZ2

[5] R. P. L. Nijssen, Fatigue life prediction and strength degradation of wind turbine rotor blade composites, Tech. Rep. SAND2006-7810P, Sandia National Laboratories.

[6] N. Dalili, A. Edrisy, R. Carriveau, A review of surface engineering issues critical to wind turbine performance, Renewable and Sustainable Energy Reviews 13 (2) (2009) 428-438. 
[7] H. Macdonald, D. Infield, D. H. Nash, M. M. Stack, Mapping hail meteorological observations for prediction of erosion in wind turbines, Wind Energy (2015) (In press).

[8] H. J. Sutherland, A summary of the fatigue properties of wind turbine materials, Wind energy 3 (1) (2000) 1-34.

[9] A. Sareen, C. A. Sapre, M. S. Selig, Effects of leading edge erosion on wind turbine blade performance, Wind Energy 17 (10) (2014) $1531-1542$.

[10] Y. Uematsu, M. Yamada, Effects of aspect ratio and surface roughness on the time-averaged aerodynamic forces on cantilevered circular cylinders at high reynolds numbers, Journal of wind engineering and industrial aerodynamics 54 (1995) 301-312.

[11] W. Chakroun, I. Al-Mesri, S. Al-Fahad, Effect of surface roughness on the aerodynamic characteristics of a symmetrical airfoil, Wind Engineering 28 (5) (2004) 547-564.

[12] G. Matteoni, C. T. Georgakis, Effects of surface roughness and cross-sectional distortion on the wind-induced response of bridge cables in dry conditions, Journal of Wind Engineering and Industrial Aerodynamics 136 (2015) 89-100.

[13] F. Schuerich, R. E. Brown, Effect of dynamic stall on the aerodynamics of vertical-axis wind turbines, AIAA journal 49 (11) (2011) 25112521 .

[14] K. Almohammadi, D. Ingham, L. Ma, M. Pourkashanian, Modeling dynamic stall of a straight blade vertical axis wind turbine, Journal of Fluids and Structures 57 (2015) 144-158.

[15] P. Pourazarm, L. Caracoglia, M. Lackner, Y. Modarres-Sadeghi, Stochastic analysis of flow-induced dynamic instabilities of wind turbine blades, Journal of Wind Engineering and Industrial Aerodynamics 137 (2015) 37-45.

[16] G. H. Jilbert, J. E. Field, Synergistic effects of rain and sand erosion, Wear 243 (1) (2000) 6-17.

[17] K. Wood, Blade repair: Closing the maintenance gap, Composites Technology (2011).

[18] L. Rempel, Rotor blade leading edge erosion-real life experiences, Wind Systems Magazine (2012).

[19] S. Stephenson, Wind blade repair: Planning, safety, flexibility, Composites World (2011). URL http: //goo.gl/JAuwfm

[20] P. Giguère, M. S. Selig, Aerodynamic effects of leading-edge tape on aerofoils at low reynolds numbers, Wind Energy 2 (3) (1999) 125-136.

[21] W. Yang, P. J. Tavner, C. J. Crabtree, Y. Feng, Y. Qiu, Wind turbine condition monitoring: technical and commercial challenges, Wind Energy 17 (5) (2014) 673-693.

[22] B. Amirzadeh, A. Louhghalam, M. Raessi, M. Tootkaboni, A computational framework for rain erosion prediction in wind turbine blades, part I: stochastic rain texture model and raindrop impact simulations, Journal of Wind Engineering \& Industrial Aerodynamics (Under review).

[23] D. T. Griffith, T. D. Ashwill, The sandia 100-meter all-glass baseline wind turbine blade: SNL100-00, Tech. rep., Sandia National Laboratories (2011).

[24] W. Hu, K. Choi, O. Zhupanska, J. H. Buchholz, Integrating variable wind load, aerodynamic, and structural analyses towards accurate fatigue life prediction in composite wind turbine blades, Structural and Multidisciplinary Optimization 53 (3) (2016) 375-394.

[25] D. S. Simulia, ABAQUS Documentation (2013).

[26] B. Bhuvaraghan, S. M. Srinivasan, B. Maffeo, R. D. McCLain, Y. Potdar, O. Prakash, Shot peening simulation using discrete and finite element methods, Advances in Engineering Software 41 (12) (2010) 1266-1276.

[27] R. Ghelichi, S. Bagherifard, M. Guagliano, M. Verani, Numerical simulation of cold spray coating, Surface and Coatings Technology 205 (23) (2011) 5294-5301.

[28] P. Gudimetla, P. Yarlagadda, Finite element analysis of the interaction between an AWJ particle and a polycrystalline alumina ceramic, Journal of Achievements in Materials and Manufacturing Engineering 23 (1) (2007) 7-14.

[29] E. Yarrapareddy, R. Kovacevic, Numerical simulation and characterization of slurry erosion of laser cladded surfaces by using failure analysis approach, Journal of Failure Analysis and Prevention 7 (6) (2007) 464-474.

[30] Z. Na, Y. Fan, L. Lei, S. Changyu, J. Castro, L. James, Thickness effect on particle erosion resistance of thermoplastic polyurethane coating on steel substrate, Wear 303 (12) (2013) 49 - 55.

[31] M. Bielawski, W. Beres, FE modelling of surface stresses in erosion-resistant coatings under single particle impact, Wear 262 (1) (2007) 
$167-175$

[32] M. S. ElTobgy, E. Ng, M. A. Elbestawi, Finite element modeling of erosive wear, International Journal of Machine Tools and Manufacture 45 (11) (2005) 1337-1346.

[33] Q. Zhou, N. Li, X. Chen, T. Xu, S. Hui, D. Zhang, Liquid drop impact on solid surface with application to water drop erosion on turbine blades, part ii: Axisymmetric solution and erosion analysis, International Journal of Mechanical Sciences 50 (10) (2008) 1543-1558.

[34] M. H. Keegan, D. H. Nash, M. M. Stack, Modelling rain drop impact of offshore wind turbine blades, in: ASME Turbo Expo 2012: Turbine Technical Conference and Exposition, American Society of Mechanical Engineers, 2012, pp. 887-898.

[35] G. Prayogo, H. Homma, T. P. Soemardi, A. S. Danardono, Impact fatigue damage of GFRP materials due to repeated raindrop collisions, Transactions of the Indian Institute of Metals 64 (4-5) (2011) 501-506.

[36] M. K. Lee, W. W. Kim, C. K. Rhee, W. J. Lee, Investigation of liquid impact erosion for 12Cr steel and Stellite 6b, Journal of Nuclear Materials 257 (2) (1998) 134-144.

[37] A. Thiruvengadam, S. L. Rudy, M. Gunasekaran, Experimental and analytical investigations on liquid impact erosion, Characterization and Determination of Erosion Resistance, ASTM STP 474 (1970) 249-287.

[38] J. E. Field, ELSI conference: Invited lecture liquid impact: Theory, experiment, applications 233-235 (1999) 1-12.

[39] G. S. Springer, C. B. Baxi, A model for rain erosion of homogenous materials, STP 567 (1974) 106-127.

[40] C. M. Preece, N. H. Macmillan, Erosion, Annual Review of Materials Science 7 (1) (1977) 95-121.

[41] H. M. Slot, E. R. M. Gelinck, C. Rentrop, E. van der Heide, Leading edge erosion of coated wind turbine blades: Review of coating life models, Renewable Energy 80 (2015) 837-848.

[42] G. P. Thomas, J. H. Brunton, Drop impingement erosion of metals, Proceedings of the Royal Society of London. A. Mathematical and Physical Sciences 314 (1519) (1970) 549-565.

[43] S. Hattori, T. Hirose, K. Sugiyama, Prediction method for cavitation erosion based on measurement of bubble collapse impact loads, Wear 269 (7) (2010) 507-514.

[44] N. W. M. Bishop, F. Sherratt, Finite element based fatigue calculations, National Agency for Finite Element Methods and Standards, Hamilton, Scotland, 2000

[45] S. D. Downing, D. F. Socie, Simple rainflow counting algorithms, International Journal of Fatigue 4 (1) (1982) 31-40.

[46] Y. J. Jang, C. W. Choi, J. H. Lee, K. W. Kang, Development of fatigue life prediction method and effect of 10-minute mean wind speed distribution on fatigue life of small wind turbine composite blade, Renewable Energy 79 (2015) 187-198.

[47] P. Brøndsted, R. P. L. Nijssen, Advances in wind turbine blade design and materials, Elsevier, Amsterdam, Netherlands, 2013.

[48] Y. Liu, S. Mahadevan, Probabilistic fatigue life prediction of multidirectional composite laminates, Composite Structures 69 (1) (2005) 11-19.

[49] W. Hu, K. Choi, H. Cho, Reliability-based design optimization of wind turbine blades for fatigue life under dynamic wind load uncertainty, Structural and Multidisciplinary Optimization 54 (4) (2016) 953-970. 